Article

\title{
The Existence of Two Homogeneous Geodesics in Finsler Geometry
}

\author{
Zdeněk Dušek \\ Institute of Technology and Business in České Budějovice, Okružní 517/10, \\ 37001 České Budějovice, Czech Republic; zdusek@mail.vstecb.cz
}

Received: 31 May 2019; Accepted: 24 June 2019; Published: 1 July 2019

\begin{abstract}
The existence of a homogeneous geodesic in homogeneous Finsler manifolds was positively answered in previous papers. However, the result is not optimal. In the present paper, this result is refined and the existence of at least two homogeneous geodesics in any homogeneous Finsler manifold is proved. In a previous paper, examples of Randers metrics which admit just two homogeneous geodesics were constructed, which shows that the present result is the best possible.
\end{abstract}

Keywords: homogeneous manifold; homogeneous Finsler space; homogeneous geodesic

MSC: 53C22; 53C60; 53C30

\section{Introduction}

Homogeneous spaces are a natural generalization of symmetric spaces and they keep many of their nice properties. One of them is the existence of a transitive group of transformations, which are sometimes called symmetries. The importance of geodesic curves is well known in mathematics and also in physics and homogeneous geodesics are, moreover, orbits of these symmetries. In physics, they are related with relative equilibria. In Riemannian geometry, homogeneous geodesics were studied by many authors and many results were obtained, see the recent survey paper [1] by the author. In recent years, homogeneous geodesics attained interest in Finsler geometry. In the present paper, we shall focus on the existence of homogeneous geodesics in homogeneous Finsler manifolds and on an interesting phenomenon related with nonreversibility of general Finsler metrics and consequent nonreversibility of homogeneous geodesics.

The existence of at least one homogeneous geodesic in arbitrary homogeneous Riemannian manifold was proved by O. Kowalski and J. Szenthe in [2]. In the papers [3,4], it was proved that this result is optimal, namely, examples of homogeneous Riemannian metrics on solvable Lie groups were constructed which admit just one homogeneous geodesic through any point. Generalization of this existence result to pseudo-Riemannian geometry was proved by the author using a different approach in the broader context of homogeneous affine manifolds in [5]. This affine approach was used by the author also in [6] to prove that an even-dimensional Lorentzian manifold admits a light-like homogeneous geodesic.

Generalization of this existence result to Finsler geometry was proved in the series of papers [7] by Z. Yan and S. Deng for Randers metrics, [8] by the author for odd-dimensional Finsler metrics, [9] by the author for Berwald or reversible Finsler metrics, [10] by Z. Yan and L. Huang in general. In this last paper, an original approach by O. Kowalski and J. Szenthe is modified and a purely Finslerian construction is used. However, due to the nonreversibility of general Finsler metrics, it was conjectured by the author in [11] that the result and its proofs in the nonreversible situation are not optimal. In comparison with Riemannian geometry, the situation is rather delicate. In the context of Finsler geometry, the trajectory of the unique homogeneous geodesic in a Riemannian manifold should be 
regarded as two geodesics-they have the same trajectory, their initial vectors are $X$ and $-X$ and they have opposite parametrizations. For a general homogeneous Finsler manifold, the initial vectors of the two homogeneous geodesics may be non-opposite. In the paper [11], examples of invariant Randers metrics which admit just two homogeneous geodesics were constructed. The initial vectors of these geodesics are $X+Y$ and $-X+Y$, for certain vectors $X, Y \in T_{p} M$.

In the present paper, the mentioned proofs are revised and refined. The complete and selfcontained proof of the existence of two homogeneous geodesics through an arbitrary point in arbitrary homogeneous Finsler manifold is given. Some constructions from $[2,10,12]$ are used.

\section{Basic Settings}

A Minkowski norm on the vector space $\mathbb{V}$ is a nonnegative function $F: \mathbb{V} \rightarrow \mathbb{R}$ which is smooth on $\mathbb{V} \backslash\{0\}$, positively homogeneous $(F(\lambda y)=\lambda F(y)$ for any $\lambda>0)$ and whose Hessian $g_{i j}=\left(\frac{1}{2} F^{2}\right)_{y^{i} y j}$ is positively definite on $\mathbb{V} \backslash\{0\}$. Variables $\left(y^{i}\right)$ are the components of a vector $y \in \mathbb{V}$ with respect to a basis $B$ of $\mathbb{V}$ and putting $y^{i}$ to a subscript refers to the partial derivative. The pair $(\mathbb{V}, F)$ is called a Minkowski space. The tensor $g_{y}$ whose components are $g_{i j}(y)$ is the fundamental tensor. We recall the well known formulas

$$
\begin{aligned}
& g_{y}(y, u)=\left.\frac{1}{2} \frac{d F^{2}(y+s u)}{d s}\right|_{s=0^{\prime}} \quad \forall y, u \in \mathbb{V}, \\
& g_{y}(y, y)=F^{2}(y), \quad \forall y \in \mathbb{V} .
\end{aligned}
$$

A Finsler metric on a differentiable manifold $M$ is a function $F$ on $T M$ which is differentiable on $T M \backslash\{0\}$ and such that its restriction to any tangent space $T_{x} M$ is a Minkowski norm. The pair $(M, F)$ is called a Finsler manifold. On a Finsler manifold, functions $g_{i j}$ depend differentiably on $x \in M$ and on $o \neq y \in T_{x} M$.

Let $M$ be a Finsler manifold $(M, F)$. If some connected Lie group $G$ acts transitively on $M$ by isometries, then $M$ is called a homogeneous manifold. We remark that a homogeneous manifold $(M, F)$ may admit more presentations as a homogeneous space in the form $G / H$, corresponding to various transitive isometry groups.

Homogeneous manifold $M$ can be identified with the homogeneous space $G / H$. Here $H$ is the isotropy group of the origin $p \in M$. A homogeneous Finsler space $(G / H, F)$ is a reductive homogeneous space in the following sense: Denote by $\mathfrak{g}$ and $\mathfrak{h}$ the Lie algebras of the groups $G$ and $H$, respectively, and consider the representation $\mathrm{Ad}: H \times \mathfrak{g} \rightarrow \mathfrak{g}$ of $H$ on $\mathfrak{g}$. There exists a reductive decomposition $\mathfrak{g}=\mathfrak{m}+\mathfrak{h}$ where $\mathfrak{m} \subset \mathfrak{g}$ is a vector subspace with the property $\operatorname{Ad}(H)(\mathfrak{m}) \subset \mathfrak{m}$. For a fixed reductive decomposition $\mathfrak{g}=\mathfrak{m}+\mathfrak{h}$ it is natural to identify $\mathfrak{m} \subset \mathfrak{g}=T_{e} G$ with the tangent space $T_{p} M$ via the projection $\pi: G \rightarrow G / H=M$. Using this identification, from the Minkovski norm and its fundamental tensor on $T_{p} M$, we obtain the $\operatorname{Ad}(H)$-invariant Minkowski norm and the $\operatorname{Ad}(H)$-invariant fundamental tensor on $\mathfrak{m}$.

We further recall the slit tangent bundle $T M_{0}$, which is defined as $T M_{0}=T M \backslash\{0\}$. Using the restriction of the projection $\pi: T M \rightarrow M$ to $T M_{0}$, we construct the pullback vector bundle $\pi^{*} T M$ over $T M_{0}$. The Chern connection is the unique linear connection on $\pi^{*} T M$ which is torsion free and almost g-compatible. See some monograph, for example [13] by D. Bao, S.-S. Chern and Z. Shen or [14] by S. Deng for details. Using the Chern connection, the derivative along a curve $\gamma(t)$ can be defined. A regular differentiable curve $\gamma$ with tangent vector field $T$ is a geodesic if it holds $D_{T}\left(\frac{T}{F(T)}\right)=0$. In particular, for a geodesic of constant speed it holds $D_{T} T=0$.

A geodesic $\gamma(s)$ through the point $p$ is homogeneous if it is an orbit of a one-parameter group of isometries. Explicitly, if there exists a nonzero vector $X \in \mathfrak{g}$ such that $\gamma(t)=\exp (t X)(p)$ for all $t \in \mathbb{R}$. Such a vector $X$ is called a geodesic vector. Geodesic vectors are characterized by the geodesic lemma, proved in Riemannian geometry by O. Kowalski and L. Vanhecke in [15] and generalized to Finsler geometry by D. Latifi in [16]. 
Lemma 1 ( [16]). Let $(G / H, F)$ be a homogeneous Finsler space with a reductive decomposition $\mathfrak{g}=\mathfrak{m}+\mathfrak{h}$. A nonzero vector $y \in \mathfrak{g}$ is geodesic if and only if it holds

$$
g_{y_{\mathfrak{m}}}\left(y_{\mathfrak{m}},[y, u]_{\mathfrak{m}}\right)=0 \quad \forall u \in \mathfrak{m},
$$

where the subscript $\mathfrak{m}$ indicates the projection of a vector from $\mathfrak{g}$ to $\mathfrak{m}$.

\section{The Main Result}

Theorem 1. Let $(M, F)$ be a homogeneous Finsler manifold. There exist at least two homogeneous geodesics through arbitrary point $p \in M$.

Proof. Let $G$ be a transitive isometry group of $M$ and let $H$ be the isotropy group of a fixed point $p \in M$. We express $M$ as the homogeneous space $M=G / H$. Let $K$ be the Killing form on $G$ and let $\operatorname{Rad}(K)$ be the null space of $K$. We choose $\mathfrak{m}=\mathfrak{h}^{\perp}$ with respect to $K$. The decomposition in $\operatorname{Ad}(H)$-invariant and the Finsler metric induces the invariant Minkowski norm and its fundamental tensor on $\mathfrak{m}$. We shall denote these again by $F$ and $g$. The Killing form $K$ is negatively semidefinite on $\mathfrak{g}$ and negatively definite on $\mathfrak{h}$, because $H$ is compact. Hence, $\operatorname{Rad}(K) \subseteq \mathfrak{m}$. We shall distinguish the two cases:

(Case 1) $\operatorname{Rad}(K)=\mathfrak{m}$ : we chose a hyperplane $W \subset \mathfrak{m}$ such that $[\mathfrak{m}, \mathfrak{m}] \subset W$. We used the construction and notation from [12] to show that there exist two vectors $n_{1}, n_{2} \in \mathfrak{m}$ such that

$$
g_{n_{i}}\left(n_{i}, w\right)=0 \quad \forall w \in W, \quad i=1,2 .
$$

Consider an arbitrary fixed vector $v \notin W$. The function $\phi(w):=F(v-w)$ defined on $W$ attains its minimum $m$ at a unique point $w_{0} \in W$. We put

$$
n_{1}=\frac{v-w_{0}}{m}
$$

It can be proved that the definition of the vector $n_{1}$ does not depend on the choice of the vector $v$ on the same side of the hyperplane $W$. If we start with a vector $v$ on the other side of the hyperplane $W$, the same construction leads to the vector $n_{2}$ on the other side of the hyperplane $W$ and it is in general not opposite to $n_{1}$, unless $F$ is reversible. We shall now write $n$ for any of the two vectors $n_{1}, n_{2}$. For an arbitrary fixed vector $w \in W$, the equality

$$
F^{2}(n+t w)=\frac{1}{m^{2}} F^{2}\left(v-w_{0}+t m w\right)=\frac{1}{m^{2}} \phi^{2}\left(w_{0}-t m w\right),
$$

shows that the function $F^{2}(n+t w)$ attains its minimum at $t=0$ and hence, using Formula (1), it holds

$$
0=\left.\frac{1}{2} \frac{d}{d t} F^{2}(n+t w)\right|_{t=0}=g_{n}(n, w), \quad \forall w \in W,
$$

which is the desired property. In particular, it is satisfied for any $w \in[\mathfrak{m}, \mathfrak{m}] \subset W$. We obtain immediately, using Lemma 1 , that $n_{1}$ and $n_{2}$ are geodesic vectors.

(Case 2) $\operatorname{Rad}(K) \subsetneq \mathfrak{m}$ : we started with the construction and notation as in [10], up to a sign. We shall investigate the function

$$
f(z)=-\frac{K(z, z)}{F^{2}(z)}
$$


which is nonnegative on $\mathfrak{m} \backslash\{0\}$. This function is homogeneous and it is reasonable to restrict the definition domain to the indicatrix

$$
I_{F}=\{z \in \mathfrak{m} ; F(z)=1\} .
$$

The function $f(z)$ attains its maximum $\lambda_{1}$ at $y_{1} \in I_{F}$. To find the second vector is more delicate. Since the group $H$ is compact and $\operatorname{Rad}(K)$ is an $\operatorname{Ad}(H)$-invariant subspace, there exists an $\operatorname{Ad}(H)$-invariant $K$-orthogonal complement $W$ of $\operatorname{Rad}(K)$ in $\mathfrak{m}$. Each vector $z \in \mathfrak{m}$ can be uniquely decomposed as $z=z_{1}+z_{2}$, where $z_{1} \in \operatorname{Rad}(K)$ and $z_{2} \in W$. Denote $k=\operatorname{dim}(\operatorname{Rad}(K))$ and let

$$
D_{k}=\left\{z_{1} \in \operatorname{Rad}(K), F\left(z_{1}\right)<1\right\}
$$

be the open unit disc in $\operatorname{Rad}(K)$. For each fixed $z_{1} \in D_{k}$, consider the set

$$
S_{z_{1}}=\left\{z_{2} \in W, F\left(z_{1}+z_{2}\right)=1\right\},
$$

which has the topology of a sphere. From now on, if not stated otherwise, $z_{1}+z_{2}$ means $z_{1} \in D_{k}$, $z_{2} \in S_{z_{1}}$ and $z_{1}+z_{2} \in I_{F}$. Because $-K>0$ on $W$, the function $f\left(z_{1}+z_{2}\right)$ is positive for any $z_{1} \in D_{k}$ and $\lim _{z_{1} \rightarrow \partial D_{k}} f\left(z_{1}+z_{2}\right)=0$. For fixed $z_{1}$ and with definition domain $S_{z_{1}}, f\left(z_{1}+z_{2}\right)$ attains its minimum $\varepsilon\left(z_{1}\right)>0$ at some $\bar{z}_{2}\left(z_{1}\right) \in S_{z_{1}}$. For each $z_{1} \in D_{k}$, we choose one such $\bar{z}_{2}$ and consider the mapping $\varphi: D_{k} \rightarrow I_{F}, z_{1} \mapsto z_{1}+\bar{z}_{2}$. The function $f\left(\varphi\left(z_{1}\right)\right)=\varepsilon\left(z_{1}\right)$ is smooth on $D_{k}$ and it attains its maximum $\lambda_{2}$ at $\bar{z}_{1}$. Here $\bar{z}_{1}$ can be chosen and the map $\varphi$ can be defined in a way that there is a neighbourhood $U \subset D_{k}$ of $\bar{z}_{1}$ such that the mapping $\left.\varphi\right|_{U}$ is smooth. We put $y_{2}=\varphi\left(\bar{z}_{1}\right) \in I_{F}$.

It remains to show that $y_{1}$ and $y_{2}$ are geodesic vectors. As to $y_{1}$, the function

$$
\tilde{f}(z)=K(z, z)+\lambda_{1} F^{2}(z)
$$

attains its minimum 0 at $y_{1}$. For any fixed $w \in \mathfrak{m}$, the function $\hat{f}(t)=\tilde{f}\left(y_{1}+t w\right)$ attains its minimum 0 at $t=0$ and hence $\hat{f}^{\prime}(0)=0$. Using Formula (1), it follows that

$$
-K\left(y_{1}, w\right)=\lambda_{1} \cdot g_{y_{1}}\left(y_{1}, w\right), \quad \forall w \in \mathfrak{m}
$$

and the formula

$$
g_{y_{1}}\left(y_{1},\left[y_{1}, z\right]_{\mathfrak{m}}\right)=\frac{-1}{\lambda} K\left(y_{1},\left[y_{1}, z\right]_{\mathfrak{m}}\right)=\frac{-1}{\lambda} K\left(\left[y_{1}, y_{1}\right], z\right)=0, \quad \forall z \in \mathfrak{m}
$$

shows that $y_{1}$ is a geodesic vector. As to $y_{2}$, we have to modify this approach. The function

$$
\tilde{f}(z)=K(z, z)+\lambda_{2} F^{2}(z)
$$

attains value 0 at $y_{2}$. For fixed $u \in W$, the function $\hat{f}(t)=\tilde{f}\left(y_{2}+t u\right)$ attains its maximum 0 at $t=0$ and hence $\hat{f}^{\prime}(0)=0$. It follows that

$$
-K\left(y_{2}, u\right)=\lambda_{2} \cdot g_{y_{2}}\left(y_{2}, u\right), \quad \forall u \in W .
$$

Now, let $v \in \operatorname{Rad}(K)$ be arbitrary fixed vector. Recall that $y_{2}=z_{1}+z_{2}$. Consider the line $z_{1}+t v$ in $\operatorname{Rad}(K)$, the curve $c(t)=\varphi\left(z_{1}+t v\right)$ in $I_{F}$ and denote by $\bar{v}$ the tangent vector to $c(t)$ at $t=0$. The function $\hat{f}(t)=\tilde{f}(c(t))$ attains its minimum 0 at $t=0$ and hence $\hat{f}^{\prime}(0)=0$. It follows that

$$
-K\left(y_{2}, \overline{\mathcal{v}}\right)=\lambda_{2} \cdot g_{y_{2}}\left(y_{2}, \overline{\mathcal{v}}\right) .
$$


Consider a basis $\left\{u_{i}\right\}$ of $W$, a basis $\left\{v_{j}\right\}$ of $\operatorname{Rad}(K)$ and construct vectors $\bar{v}_{j}$ as above. It is easy to see that $\left\{u_{i}, \bar{v}_{j}\right\}$ is a basis of $\mathfrak{m}$ and hence Formulas (2) and (3) for each vector $\bar{v}_{j}$ imply

$$
-K\left(y_{2}, w\right)=\lambda_{2} \cdot g_{y_{2}}\left(y_{2}, w\right), \quad \forall w \in \mathfrak{m} .
$$

We finish the proof with the formula

$$
g_{y_{2}}\left(y_{2},\left[y_{2}, z\right]_{\mathfrak{m}}\right)=\frac{-1}{\lambda_{2}} K\left(y_{2},\left[y_{2}, z\right]_{\mathfrak{m}}\right)=\frac{-1}{\lambda_{2}} K\left(\left[y_{2}, y_{2}\right], z\right)=0, \quad \forall z \in \mathfrak{m},
$$

which shows that $y_{2}$ is a geodesic vector.

Funding: Research was funded by Internal Grant Agency of Institute of Technology and Business in České Budějovice grant number IGS 8210-009.

Conflicts of Interest: The author declares no conflict of interest. The funders had no role in the design of the study; in the collection, analyses, or interpretation of data; in the writing of the manuscript, or in the decision to publish the results.

\section{References}

1. Dušek, Z. Homogeneous geodesics and g.o. manifolds. Note Mat. 2018, 38, 1-15.

2. Kowalski, O.; Szenthe, J. On the existence of homogeneous geodesics in homogeneous Riemannian manifolds. Geom. Dedicata 2000, 81, 209-214; Erratum: Geom. Dedicata 2001, 84, 331-332. [CrossRef]

3. Kowalski, O.; Nikčević, S. and Vlášek, Z. Homogeneous Geodesics in Homogeneous Riemannian Manifolds_Examples; Preprint Reihe Mathematik, TU Berlin, No. 665/2000; TU Berlin: Berlin, Germany, 2000.

4. Kowalski, O.; Vlášek, Z. Homogeneous Riemannian manifolds with only one homogeneous geodesic. Publ. Math. Debrecen 2003, 62, 437-446.

5. Dušek, Z. The existence of homogeneous geodesics in homogeneous pseudo-Riemannian and affine manifolds. J. Geom. Phys. 2010, 60, 687-689. [CrossRef]

6. Dušek, Z. The existence of light-like homogeneous geodesics in homogeneous Lorentzian manifolds. Math. Nachr. 2015, 288, 872-876. [CrossRef]

7. Yan, Z.; Deng, S. Existence of homogeneous geodesics on homogeneous Randers spaces. Houston J. Math. 2018, 44, 481-493.

8. Dušek, $Z$. The affine approach to homogeneous geodesics in homogeneous Finsler spaces. Archivum Mathematicum (Brno) 2018, 54, 127-133. [CrossRef]

9. Dušek, Z. The existence of homogeneous geodesics in special homogeneous Finsler spaces. Matematički Vesnik 2019, 71, 16-22.

10. Yan, Z.; Huang, L. On the existence of homogeneous geodesic in homogeneous Finsler spaces. J. Geom. Phys. 2018, 124, 264-267. [CrossRef]

11. Dušek, Z. Homogeneous Randers spaces admitting just two homogeneous geodesics. Archivum Mathematicum (Brno) 2019, in press.

12. Shen, Z. Lectures on Finsler Geometry; World Scientific: Singapore, 2001.

13. Bao, D.; Chern, S.-S.; Shen, Z. An Introduction to Riemann-Finsler Geometry; Springer Science+Business Media: New York, NY, USA, 2000.

14. Deng, S. Homogeneous Finsler Spaces; Springer Science+Business Media: New York, NY, USA, 2012.

15. Kowalski, O.; Vanhecke, L. Riemannian manifolds with homogeneous geodesics. Boll. Un. Math. Ital. 1991, 5, 189-246.

16. Latifi, D. Homogeneous geodesics in homogeneous Finsler spaces. J. Geom. Phys 2007, 57, 1421-1433. [CrossRef]

(C) 2019 by the author. Licensee MDPI, Basel, Switzerland. This article is an open access article distributed under the terms and conditions of the Creative Commons Attribution (CC BY) license (http:/ / creativecommons.org/licenses/by/4.0/). 